Proceedings

\title{
Sensing Characteristics of Smart Microsensor Systems for Measuring Relative Humidity in Pressurized Air ${ }^{\dagger}$
}

\author{
Fabian Weller ${ }^{1}$, Dino Keller ${ }^{1}$, Sascha Wettstein ${ }^{2}$ and Markus Graf ${ }^{1, *}$ \\ 1 Sensirion AG, Laubisruetistrasse 50, 8712 Staefa, Switzerland; fabian.weller@sensirion.com (F.W.); \\ dino.keller@sensirion.com (D.K.) \\ 2 MBW Calibration AG, Seminarstrasse 55/57, 5430 Wettingen, Switzerland; sascha@mbw.ch (S.W.) \\ * Correspondence: markus.graf@sensirion.com; Tel.: +41-44-306-4944 \\ + Presented at the Eurosensors 2018 Conference, Graz, Austria, 9-12 September 2018.
}

Published: 19 December 2018

\begin{abstract}
The detailed experimental investigation of the performance of a commercially available smart humidity sensor used for the measurement of relative humidity at elevated pressures (0.1-2 $\mathrm{MPa}$ ) is reported. A dedicated set-up was developed for generating defined humidities in pressurized air. The measurements reveal that the pressure coefficient of the sensor deviation at a given relative humidity condition cannot be attributed solely to the enhancement factor of the humid air. For such microsensors, an additional sensor-specific pressure compensation factor was determined which is significant for precise measurements in applications with pressurized air.
\end{abstract}

Keywords: humidity sensor; enhancement factor

\section{Introduction}

Novel applications of smart environmental sensors are emerging in the fields of automotive, internet of things, and future manufacturing, fostered by their miniaturization. Measuring and controlling relative humidity is important for all these applications in order to improve energy efficiency, safety and comfort. Polymer-based humidity microsensors are the standard for such applications and their characteristics at ambient conditions are well understood. However, under elevated pressures $(>0.1 \mathrm{MPa})$ there is still a lack of reliable measurements. The correct determination of relative humidity under pressure is commonly described by the enhancement factor [1,2]. At pressures close to ambient conditions, these corrections are negligible compared to typical sensor accuracies. However, Stevens et al. (2010) [3] indicated that for polymer-based humidity sensors the measured values cannot be fully explained with the enhancement factor. They revealed an additional contribution, which is associated with the transduction principle. These effects have not yet been investigated, despite their usefulness in determining humidity in pressurized systems e.g., in natural gas or industrial pipes. Therefore, we have built a dedicated set-up for generating defined relative humidity set points at different pressures and temperatures for advanced measurements. With this set-up, we have for the first time systematically determined this additional sensor-specific pressure compensation factor for a commercially available smart humidity sensor. 


\section{Measurement of Relative Humidity in Pressurized Air}

The definition of relative humidity $U^{\prime}{ }_{w}$ under pressurized conditions is described as:

$$
U^{\prime}{ }_{W}=\frac{e}{e^{\prime}{ }_{w}(p, t)}=\frac{e}{f_{w}(p, t) \cdot e_{w}(p, t)}=\frac{U_{W}}{f_{w}(p, t)}
$$

where $e$ corresponds to the partial water pressure, $t$ is the temperature in ${ }^{\circ} \mathrm{C}, e^{\prime} w$ represents the saturation vapour pressure including the dependence on the total pressure $p$ by the so-called enhancement factor $f_{w}(p, t), p$ is the sum of the partial water pressure and the dry carrier gas pressure pdry:

$$
p=e+p_{d r y}
$$

and $e_{w}$ is the saturation vapour pressure generated in equilibrium above liquid water. Thus, $U_{w}$ corresponds to the relative humidity excluding gas pressure effects. For values $t<0$ the formula must be adjusted to vapour pressures over ice. The enhancement factor has been measured for a wide range of temperatures and pressures and respective formulae are summarized in Greenspan [1] and modernized by Hardy [2].

Commercially available humidity sensors are calibrated at ambient pressures of $\sim 0.1 \mathrm{MPa}$ and any modifications by the enhancement factors for atmospheric pressure changes are small compared to typical accuracies in sensor technology, of e.g., $2 \%$ RH. However, for applications under higher pressures the contribution becomes more significant and for precise measurements has to be taken into account. For example, an additional pressure of $2 \mathrm{bar}(0.2 \mathrm{MPa})$ generates for a relative humidity of $50 \% \mathrm{RH}$ (at $25{ }^{\circ} \mathrm{C}$ and atmospheric pressure of $0.1 \mathrm{MPa}$ ) a contribution of $-0.35 \% \mathrm{RH}$ compared to a value not including the effect of the enhancement factor.

Apart from this thermophysical effect, an additional pressure dependence might arise from the transduction principle. For example, if a polymeric sensitive material is used, there is a partitioning coefficient between the partial water vapour pressure outside and inside the polymer. Overall pressure might have an influence on this partitioning coefficient resulting in a pressure-dependent sensor signal. Other effects on the overall sensor system might generate additional pressuredependent contributions. They have to be thoroughly investigated to achieve precise sensor measurements under pressurized conditions and to extract an additional sensor-specific pressure compensation factor $f_{s c}$. Accordingly, Equation (1) can be modified to:

$$
U^{\prime}{ }_{W}=\frac{U_{W}}{f_{w}(p, t) \cdot f_{S C}(p, t)}
$$

where the factor $f_{s c}$ may also have a temperature dependence.

\section{Materials and Methods}

\subsection{Sensors Under Test}

The measurements were performed using a commercially available Sensirion SHT31, which is a polymer-based capacitive humidity sensor [4]. The sensor element consists of an in-plane capacitor based on inter-digitated finger electrodes. The dielectric of this capacitor is a polymer on top of the electrodes, which absorbs or releases water in proportion to the relative environmental humidity, and thus changes the capacitance of the capacitor. This change in capacitance represents relative humidity and is measured by an electronic circuit. On the SHT31 sensor, the sensor element and readout electronics are combined on a single microchip. The on-chip electronics also includes linearization, temperature compensation and individual calibration data for each sensor. 


\subsection{Measurement Set-Up}

The experimental set-up is based on the "two-temperature" saturation technique: Air is saturated with water vapour at the desired dew point temperature and then heated to the temperature of the test chamber where devices under test are located. Relative humidity in the test chamber can be controlled by adjusting the saturator temperature. Figure 1 shows a schematic of the set-up. Pressurized air is supplied to a humidity generator at a pressure of $2 \mathrm{MPa}$. At the inlet of the humidity generator, incoming airflow is controlled by a mass flow valve. The pressure $p$ inside the system is controlled by a pressure regulated expansion valve at the outlet. The incoming air is saturated by a two stage system: in a pre-saturator the air is saturated at higher temperature. Then it is guided to the saturator where the air is cooled down to the temperature tsat. Excess water is condensed and drained.

This system ensures that the air is precisely saturated at temperature tsat in the range of $5{ }^{\circ} \mathrm{C}$ to $70{ }^{\circ} \mathrm{C}$. The dew point temperature of the air in the measurement chamber $t_{d}$ is equal to $t_{s a t}$. Then the humid air from the humidity generator is heated to the temperature of the thermalized test chamber test, in which test sensors are located. These are in thermal equilibrium with the test chamber and the airflow. The temperature of the chamber test is referenced by PT-100 references. At the outlet of the test chamber, the airflow is controlled by a mass flow controller and is split into two channels: one channel passes a dew point hygrometer (MBW 373LHX) as the humidity reference, the other channel is the outlet.

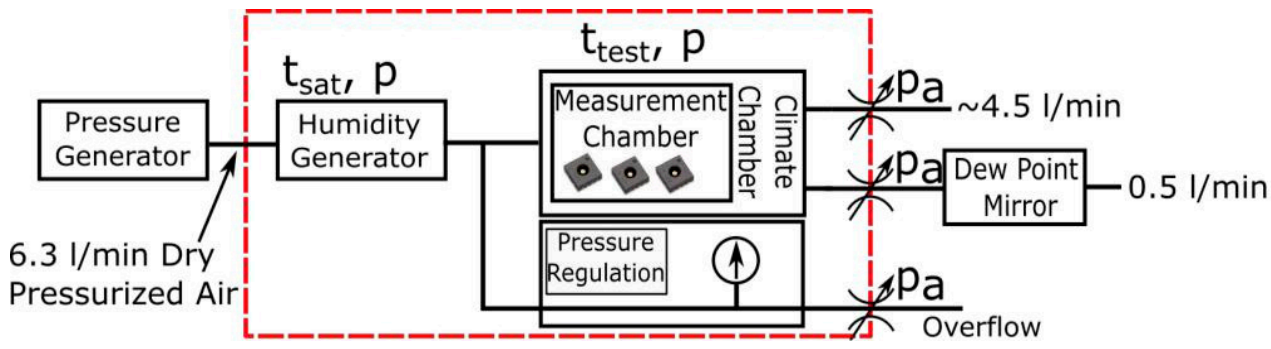

Figure 1. Schematic of measurement set-up showing the airflow from the pressure generator to the outlets. The part of the set-up inside the red dashed rectangle is pressure-controlled to pressure $\mathrm{p}$.

\subsection{Measurement Sequence}

To investigate the pressure dependence of the SHT31 the following measurement sequence was performed: The temperature of the test chamber test was stabilized at $25^{\circ} \mathrm{C}$. Then the temperature of the saturator $t_{\text {sat }}$ was chosen to reach the desired relative humidity. The airflow was switched on at the first pressure set-point, which was close to ambient pressure. The stabilization time was chosen to be long enough to minimize drifts. Subsequently, the pressure $\mathrm{p}$ was increased step by step with 30 min of dwell time. This procedure was repeated at different set-points of $t_{\text {sat. }}$

\section{Results and Discussion}

\section{Pressure-Dependence of Relative Humidity Measurements}

At varying pressure, the interaction of the atoms and molecules in a gas mixture changes, which leads to a pressure-dependence of polymer-based capacitive sensors on the saturation vapour pressure as discussed in Section 2. In our measurements, we found this pressure-dependence to be linear and roughly twice as high as described by the enhancement factor. From 0.1 to $2.0 \mathrm{MPa}$, a linear change of $4.9 \% \mathrm{RH}$ per MPa was measured at $25^{\circ} \mathrm{C}$ and a dew-point of $23.2{ }^{\circ} \mathrm{C}$, corresponding to $90 \%$ RH (see Figure 2). The correction by the enhancement factor $f_{w}$ in this figure is calculated with the formula from reference [2]. Consequently, the sensor-specific pressure-compensation factor $f_{S C}$ can be estimated from formula (3) by a linear fit to the measurement data in Figure 2 as: 


$$
f_{S C}=\frac{U_{w}}{U^{\prime}{ }_{w} \cdot f_{w}}=\frac{U_{r e f} \cdot f_{w}\left(0.1 \mathrm{MPa}, 25^{\circ} \mathrm{C}\right)}{U^{\prime}{ }_{w} \cdot f_{w}} \sim 1+0.04 \cdot\left(p-p_{a}\right)
$$

where $U_{r e f}$ is the referenced relative humidity at ambient condition and $p$ is pressure in MPa. The factor $f_{s c}$ is fixed to 1 for ambient pressure $p_{a}$ because the sensors are calibrated at ambient condition. Through further measurements at saturator temperatures of 13.9 and $6.25{ }^{\circ} \mathrm{C}$, corresponding to 50 and $30 \% \mathrm{RH}$ at $25{ }^{\circ} \mathrm{C}$ chamber temperature, respectively, we found that the effect scales with the relative humidity (see Figure 3). This is to be expected from a multiplicative factor on the effective saturation pressure. We can conclude that the factor $f_{s c}$ does not show a significant dependence on relative humidity in the limited range tested by this experiment.

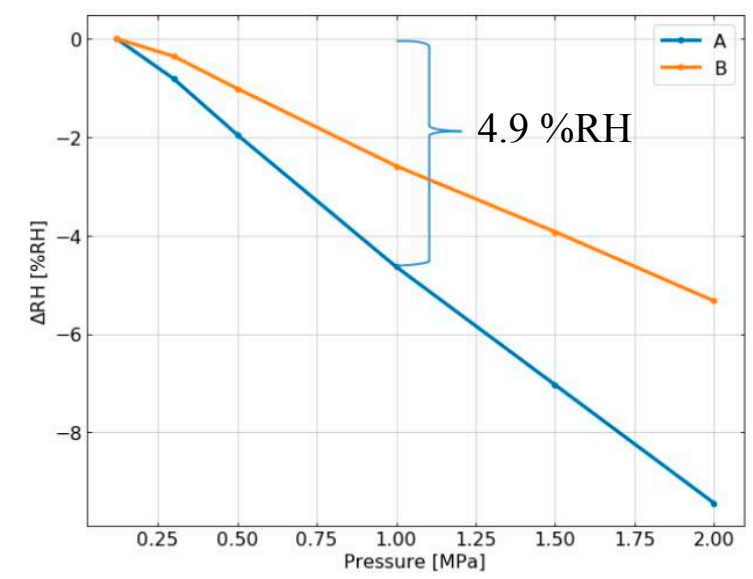

Figure 2. Measurements for an SHT 31 at a set point of $90 \% \mathrm{RH}$ at $25^{\circ} \mathrm{C}$ are plotted as a function of air pressure. Curve A shows the difference between the measured value and the reference-nulled at the first pressure set-point of $0.12 \mathrm{MPa}$. In curve B the values are corrected by the enhancement factor $f_{w}$. The remaining difference corresponds to the additional factor $f_{s c}$.

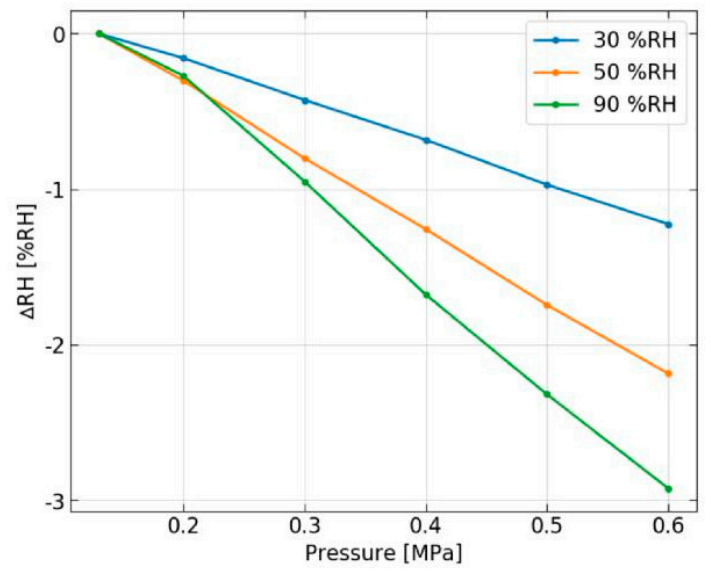

Figure 3. Measurement of the pressure dependence at different relative humidity set-points.

\section{Conclusions and Outlook}

For a precise measurement of relative humidity with polymer-based capacitive microsensors in a pressurized system, a sensor-specific pressure compensation factor $f_{S C}$ has to be introduced in addition to the enhancement factor. This factor scales linearly with pressure $p$. The origin of this additional effect remains unclear and may be the subject of further investigation. Such experiments might include measuring the temperature and relative humidity dependence of this effect and its dependence on sensor-specific properties like the material and geometry of the sensing element. 
Author Contributions: D.K. and M.G. conceived the experiments and the theoretical framework; F.W. and D.K. built the set-up, performed the experiments and analyzed the data; F.W. and S.W. performed the initial experiments; F.W., D.K. and M.G. wrote the paper.

Acknowledgments: We would like to thank Daniel Mutter (MBW Calibration AG) and Robert Benyon (INTA) for fruitful discussions and reading the manuscript.

Conflicts of Interest: The authors declare no conflict of interest.

\section{References}

1. Greenspan, L. Functional Equations for the Enhancement Factors for $\mathrm{CO}_{2}$-Free Moist Air. J. Res. Natl. Bur. Stand. A 1975, 80A, 41-44.

2. Hardy, B. ITS-90 formulations for vapor pressure, frostpoint temperature, dewpoint temperature, and enhancement factors in the range -100 to $+100 \mathrm{C}$. In Proceedings of the Third International Symposium on Humidity \& Moisture, Teddington, London, UK; 6-8 April 1998; Volume 1, pp. 206-213.

3. Stevens, M.; Bell, S.A.; Carroll, P.; Mortimer, S. Towards a standard for multi-gas and multi-pressure humidity calibrations. In Proceedings of the TEMPMEKO \& ISHM 2010, Portoroz, Slovenia, 31 May-4 June 2010; Volume A, p. 311.

4. Sensirion SHT 31. Available online: https://www.sensirion.com/en/environmental-sensors/humidity-sensors/ digital-humidity-sensors-for-various-applications/ (accessed on 21 June 2018).

(C) 2018 by the authors. Licensee MDPI, Basel, Switzerland. This article is an open access article distributed under the terms and conditions of the Creative Commons Attribution (CC BY) license (http://creativecommons.org/licenses/by/4.0/). 